\title{
MONOTONE ITERATION AND GREEN'S FUNCTIONS FOR BOUNDARY VALUE PROBLEMS
}

\author{
P. W. ELOE ${ }^{1}$ AND L. J. GRIMM ${ }^{2}$
}

\begin{abstract}
An iteration scheme is given for approximating solutions of boundary problems of the form $L y=f(x, y), T y(x)=r$, where $L$ is an $n$th order linear differential operator, $f$ is continuous and $T$ is a continuous linear operator from $C^{n-1}(I)$ into $\mathrm{R}^{n}$. The scheme is based on the condition that the Green's function $G(x, s)$ for the associated linear problem $L y=0, T y=0$ exists and has sign independent of $s$.
\end{abstract}

1. Introduction. Let $n \geqslant 1$, let $I=[a, b]$ be a real interval, let $a=x_{1}<x_{2}$ $<\cdots<x_{k}=b$, let $p_{1}(x), p_{2}(x), \ldots, p_{n}(x)$ be continuous on $I$, and define the linear differential operator $L$ by

$$
L y=y^{(n)}+p_{1}(x) y^{(n-1)}+\cdots+p_{n}(x) y .
$$

A. Ju. Levin (see [1]) has obtained the following result.

THEOREM $L$. Let $L$ and $I$ be as above, and suppose that $L$ is disconjugate on $I$. Then the Green's function $G(t, s)$ for the $k$-point boundary value problem $(B V P)$

$$
\begin{gathered}
L y=0, \\
y^{(i)}\left(x_{j}\right)=0, \quad i=0, \ldots, n_{j}-1, j=1, \ldots, k,
\end{gathered}
$$

where $\sum n_{j}=n$, satisfies the inequality

$$
G(x, s)\left(x-x_{1}\right)^{n_{1}}\left(x-x_{2}\right)^{n_{2}} \cdots \cdot\left(x-x_{k}\right)^{n_{k}}>0, \quad x_{1}<s<x_{k} .
$$

For our purposes, the importance of Levin's theorem is that in this instance the following condition holds.

Condition S. There exists a Green's function $G(x, s)$ for the problem $L y=0$, $T y=0$, and the sign of $G(x, s)$ is independent of $s$.

We present here a bilateral iteration scheme, based on Condition $\mathbf{S}$, which will provide approximants to solutions of BVP's with linear boundary conditions. A. C. Peterson has found [4] that complete disconjugacy is not necessary for this condition to hold; he has recently shown [5] that it will also hold for certain $q$-focal problems, and we shall discuss these in the last section.

2. Linear boundary value problems. Let $I$ be a real interval, let $f: I \times \mathbf{R} \rightarrow \mathbf{R}$ be continuous, and $L$ be given by (1.1). Consider the BVP

$$
L y=f(x, y)
$$

Received by the editors April 30, 1979.

1980 Mathematics Subject Classification. Primary 34B10; Secondary 34B27.

${ }^{1}$ MASUA Traveling Scholar.

${ }^{2}$ Research supported by NSF Grant MCS77-28395. 
with boundary conditions

$$
T y(x)=r,
$$

where $T: C^{n-1}(I) \rightarrow \mathbf{R}^{n}$ is a continuous linear operator, $r$ is a given constant vector. Assume that Condition $\mathbf{S}$ holds for the associated homogeneous problem $L y=0, T y(x)=0$. Hence there exist subsets of $I, I_{1}$ and $I_{2}$ such that

(i) $I=I_{1} \cup I_{2}$ (possibly $I=I_{1}$ or $I=I_{2}$ ) and

(ii) $G(x, s)$ has sign given by

$$
\begin{array}{ll}
G(x, s)<0 & \text { for } a<s<b, x \in I_{1}, \\
G(x, s)>0 & \text { for } a<s<b, x \in I_{2} .
\end{array}
$$

Assume that there exists a constant $M$ such that, for all $\left(x, y_{1}\right),\left(x, y_{2}\right)$ in $I \times \mathbf{R}$,

$$
\left|f\left(x, y_{1}\right)-f\left(x, y_{2}\right)\right|<M\left|y_{1}-y_{2}\right| \text {. }
$$

Further, suppose that there exist functions $v_{1}(x), w_{1}(x)$ with piecewise continuous $n$th derivatives on $I$, such that

$$
\begin{gathered}
T v_{1}(x)=T w_{1}(x)=r, \text { and such that, for } x \in I, \\
L v_{1}-f\left(x, v_{1}\right)+A_{1}(x) \equiv \beta_{1}(x)<0, \\
L w_{1}-f\left(x, w_{1}\right)-A_{1}(x) \equiv \gamma_{1}(x)>0,
\end{gathered}
$$

where

$$
A_{1}(x)=M\left|v_{1}(x)-w_{1}(x)\right| .
$$

Let $l_{r}(x)$ be the solution of the problem $L y=0, T y(x)=r$; existence of $l_{r}(x)$ follows from linearity and uniqueness [2]. Construct the sequences $\left\{v_{m}(x)\right\}$ and $\left\{w_{m}(x)\right\}$ as follows:

$$
\begin{aligned}
& v_{m+1}(x)=l_{r}(x)+\int_{I} G(x, s)\left[f\left(s, v_{m}(s)\right)-A_{m}(s)\right] d s, \\
& w_{m+1}(x)=l_{r}(x)+\int_{I} G(x, s)\left[f\left(s, w_{m}(s)\right)+A_{m}(s)\right] d s,
\end{aligned}
$$

where

$$
A_{m}(x)=M\left|v_{m}(x)-w_{m}(x)\right|, \quad m \geqslant 1 .
$$

Theorem 1. Let $L$ and $f$ be as above; let (2.4) and Condition $S$ hold. Suppose that there exist functions $v_{1}(x)$ and $w_{1}(x)$ satisfying (2.5), and define the sequences $\left\{v_{m}(x)\right\}$ and $\left\{w_{m}(x)\right\}$ by (2.7). Then there exists a solution $y(x)$ of the BVP (2.1)-(2.2) such that, for all $m \geqslant 1$,

$$
\begin{aligned}
& v_{m}(x) \geqslant v_{m+1}(x) \geqslant y(x) \geqslant w_{m+1}(x) \geqslant w_{m}(x), \quad x \in I_{1}, \\
& v_{m}(x)<v_{m+1}(x)<y(x) \leqslant w_{m+1}(x)<w_{m}(x), \quad x \in I_{2} .
\end{aligned}
$$

Proof. Set $u_{m}(x)=v_{m}(x)-w_{m}(x), m>1$. Note that $u_{1}(x)>0$ for $x \in I_{1}$, $u_{1}(x)<0$ for $x \in I_{2}$, since $L u_{1}=f\left(x, v_{1}\right)-f\left(x, w_{1}\right)-2 A_{1}(x)+\beta_{1}-\gamma_{1}<0$; hence $u_{1}(x)=\int_{I} G(x, s) L u_{1}(s) d s$ has sign opposite to that of $G$. The rules for constructing the sequences $\left\{v_{m}(x)\right\}$ and $\left\{w_{m}(x)\right\}$ imply, similarly, that 


$$
u_{m+1}(x)=\int_{I} G(x, s)\left[f\left(s, v_{m}(s)\right)-f\left(s, w_{m}(s)\right)-2 M\left|v_{m}(s)-w_{m}(s)\right|\right] d s
$$

and, from (2.4), we have that, for each $m \geqslant 1$,

$$
u_{m+1}(x) \geqslant-\int_{I} G(x, s) M\left|u_{m}(s)\right| d s \geqslant 0, \quad x \in I_{1},
$$

and

$$
u_{m+1}(x) \leqslant-\int_{I} G(x, s) M\left|u_{m}(s)\right| d s \leqslant 0, \quad x \in I_{2} .
$$

Hence, for each $m \geqslant 1$,

$$
v_{m} \geqslant w_{m}, \quad x \in I_{1} ; \quad v_{m} \leqslant w_{m}, \quad x \in I_{2} .
$$

To show the monotonicity of the sequences $\left\{v_{m}(x)\right\},\left\{w_{m}(x)\right\}$ on $I_{1}$ and $I_{2}$, note that $\beta_{1}=L v_{1}-L v_{2}, \gamma_{1}=L w_{1}-L w_{2}$, and set $\beta_{m}=L v_{m}-L v_{m+1}, \gamma_{m}=L w_{m}-$ $L w_{m+1}$. Using (2.11), we can write

$$
\begin{gathered}
\beta_{m+1}(x)= \begin{cases}f\left(x, v_{m}\right)-f\left(x, v_{m+1}\right)-M\left(v_{m}-v_{m+1}\right)+M\left(w_{m}-w_{m+1}\right), & x \in I_{1}, \\
f\left(x, v_{m}\right)-f\left(x, v_{m+1}\right)+M\left(v_{m}-v_{m+1}\right)-M\left(w_{m}-w_{m+1}\right), & x \in I_{2},\end{cases} \\
\gamma_{m+1}(x)= \begin{cases}f\left(x, w_{m}\right)-f\left(x, w_{m+1}\right)-M\left(w_{m}-w_{m+1}\right)+M\left(v_{m}-v_{m+1}\right), & x \in I_{1}, \\
f\left(x, w_{m}\right)-f\left(x, w_{m+1}\right)+M\left(w_{m}-w_{m+1}\right)-M\left(v_{m}-v_{m+1}\right), & x \in I_{2} .\end{cases}
\end{gathered}
$$

Setting $\delta_{m}=v_{m}-v_{m+1}, \rho_{m}=w_{m}-w_{m+1}$, we obtain, using (2.4), the inequalities

$$
\begin{array}{ll}
\beta_{m+1}(x) \leqslant M\left|\delta_{m}\right|-M \delta_{m}+M \rho_{m}, & x \in I_{1}, \\
\beta_{m+1}(x) \leqslant M\left|\delta_{m}\right|+M \delta_{m}-M \rho_{m}, & x \in I_{2} ; \\
\gamma_{m+1}(x) \geqslant-M\left|\rho_{m}\right|-M \rho_{m}+M \delta_{m}, & x \in I_{1}, \\
\gamma_{m+1}(x) \geqslant-M\left|\rho_{m}\right|+M \rho_{m}-M \delta_{m}, & x \in I_{2} .
\end{array}
$$

Since $L \rho_{1}=\gamma_{1}$ and $\gamma_{1}>0$, with $T \rho_{1}=0$, it follows that $\rho_{1}<0$ on $I_{1}, \rho_{1}>0$ on $I_{2}$. Similarly, $\delta_{1}>0$ on $I_{1}, \delta_{1}<0$ on $I_{2}$. By (2.12), $\gamma_{2}>0, \beta_{2}<0$ on $I$, and, by induction, for each $m>1, \rho_{m}<0$ on $I_{1}, \rho_{m}>0$ on $I_{2} ; \delta_{m}>0$ on $I_{1}, \delta_{m}<0$ on $I_{2}$, and $\gamma_{m}>0, \beta_{m}<0$ on all of $I$. Hence $v_{m+1}<v_{m}$ and $w_{m+1}>w_{m}$ on $I_{1}, v_{m+1}>v_{m}$ and $w_{m+1}<w_{m}$ on $I_{2}$, and we have obtained the inequalities involving the $v$ 's and $w$ 's in (2.9). It remains to show that a solution $y(x)$ lies between the $v$ 's and $w$ 's. To prove this, note first that, on $I_{1}$ and on $I_{2}$, the sequences $\left\{v_{m}(x)\right\}$ and $\left\{w_{m}(x)\right\}$ are monotonic, bounded and equicontinuous. By Ascoli's theorem, they have uniform limits $v(x)$ and $w(x)$ with $v(x)>w(x)$ for $x \in I_{1}, v(x)<w(x)$ for $x \in I_{2}$. It follows from (2.7) that

$$
L v(x)=f(x, v)-A(x), \quad L w(x)=f(x, w)+A(x),
$$

where $A(x)=M|v(x)-w(x)|$. Note that $A(x)$ is continuous and nonnegative and that $T v(x)=T w(x)=r$. 
For each function $y(x) \in C(I)$, set

$$
\bar{y}(x)=\left\{\begin{array}{ll}
v(x) & \text { if } y(x)>v(x) \\
y(x) & \text { if } v(x) \geqslant y(x) \geqslant w(x) \\
w(x) & \text { if } y(x)<w(x) \\
v(x) & \text { if } y(x)<v(x) \\
y(x) & \text { if } v(x) \leqslant y(x)<w(x) \\
w(x) & \text { if } y(x)>w(x)
\end{array}\right\}, \quad x \in I_{1},
$$

and define $\hat{F}(x, y(x))=f(x, \bar{y}(x))$. The function $\hat{F}$ is continuous and bounded on $I \times \mathbf{R}$. It follows from the Schauder fixed point theorem that the problem

$$
L y=\hat{F}(x, y), \quad T y(x)=r
$$

has a solution $y(x)$. We now show that this solution satisfies

$$
\begin{array}{ll}
v(x) \geqslant y(x) \geqslant w(x), & x \in I_{1}, \\
v(x) \leqslant y(x) \leqslant w(x), & x \in I_{2},
\end{array}
$$

and hence that $y(x)$ is a solution of (2.1)-(2.2). Let $D$ be the compact domain bounded by $v(x), w(x)$ and the lines $x=a$ and $x=b$. Set $z(x)=w(x)-y(x)$. Then

$$
\begin{aligned}
L z(x) & =L w(x)-L y(x) \\
& =f(x, w(x))+M|v(x)-w(x)|-f(x, \bar{y}(x))>0
\end{aligned}
$$

since (2.4) holds. Furthermore, since $T z(x)=0$, we have $z(x)<0, x \in I_{1}, z(x)>$ $0, x \in I_{2}$. Similarly, setting $\hat{z}(x)=v(x)-y(x)$, we obtain $\hat{z}(x)>0, x \in I_{1}, \hat{z}(x)$ $<0, x \in I_{2}$. Hence $(x, y(x))$ lies in $D$ for all $x \in I$, and the proof is complete.

REMARKS. (i) It is necessary only that the bound (2.4) hold for all $(x, y) \in D^{(1)}$, where $D^{(1)}$ is the compact domain bounded by the curves $v_{1}(x), w_{1}(x)$ and the lines $x=a$ and $x=b$.

(ii) Set $G=\max _{x \in I}\left|\int_{I} G(x, s) d s\right|$. Then if $2 M G<1$, and if $|f(x, y)|<B$, for some constant $B$ for all $(x, y) \in I \times \mathbf{R}$, the functions $v_{1}$ and $w_{1}$ can be chosen as

$$
\begin{aligned}
& v_{1}(x)=l_{r}(x)-\frac{B}{1-2 M G} \int_{I} G(x, s) d s \\
& w_{1}(x)=l_{r}(x)+\frac{B}{1-2 M G} \int_{I} G(x, s) d s
\end{aligned}
$$

(iii) In case $f$ has certain monotonicity properties, the Lipschitz continuity is not needed, and the iteration can be simplified by taking $A_{i}(x) \equiv 0$ for all $i>1$. Furthermore, the functions $v_{1}$ and $w_{1}$ can be readily obtained from $G(x, s)$ and $l_{r}(x)$ as before, but now without the requirement that $2 M G<1$. Inspection of the proof of Theorem 1 leads to the following result. 
Theorem 2. Let $L$ and $T$ be as in Theorem 1 . Let $f(x, y)$ be continuous on $I \times \mathbf{R}$, and be monotone decreasing in $y$ for each $x \in I$ and monotone increasing in $y$ for each $x \in I_{2}$. Then if there exist functions $v_{1}(x)$ and $w_{1}(x)$ satisfying

$$
\begin{gathered}
v_{1} \geqslant w_{1}, \quad x \in I_{1} ; \quad v_{1}<w_{1}, \quad x \in I_{2}, \\
T v_{1}(x)=T w_{1}(x)=r, \quad \text { and such that, for } x \in I, \\
L v_{1}-f\left(x, v_{1}\right) \equiv \beta_{1}(x)<0, \\
L w_{1}-f\left(x, w_{1}\right) \equiv \gamma_{1}(x)>0,
\end{gathered}
$$

and if the sequences $\left\{v_{m}(x)\right\},\left\{w_{m}(x)\right\}$ are defined by

$$
\begin{aligned}
& v_{m+1}(x)=l_{r}(x)+\int_{I} G(x, s) f\left(s, v_{m}(s)\right) d s, \\
& w_{m+1}(x)=l_{r}(x)+\int_{I} G(x, s) f\left(s, w_{m}(s)\right) d s,
\end{aligned}
$$

these sequences will converge to solutions $v(x)$ and $w(x)$ of the BVP (2.1)-(2.2), and

$$
\begin{array}{ll}
v_{m}(x) \geqslant v_{m+1}(x) \geqslant v(x) \geqslant w(x) \geqslant w_{m+1}(x) \geqslant w_{m}(x), & x \in I_{1}, \\
v_{m}(x) \leqslant v_{m+1}(x) \leqslant v(x)<w(x) \leqslant w_{m+1}(x)<w_{m}(x), & x \in I_{2} .
\end{array}
$$

Further, any solution $y(x)$ of the BVP (2.1)-(2.2) which lies between $v_{1}$ and $w_{1}$ will also lie between $v$ and $w$. In case $|f(x, y)|<B$ for some constant $B$ for all $(x, y) \in I \times \mathbf{R}$, the functions $v_{1}$ and $w_{1}$ can be chosen as

$$
\begin{aligned}
& v_{1}(x)=l_{r}(x)-B \int_{I} G(x, s) d s, \\
& w_{1}(x)=l_{r}(x)+B \int_{I} G(x, s) d s .
\end{aligned}
$$

3. Applications. We consider two applications.

(i) Let $L$ in (2.1) be disconjugate on $I$, and suppose that the boundary conditions (2.2) are the conjugate boundary conditions

$$
y^{(i)}\left(x_{j}\right)=c_{i j}, \quad 0<i<n_{j-1}, j=1, \ldots, k,
$$

where $\Sigma n_{j}=n, a=x_{1}<x_{2}<\cdots<x_{n}=b$, and $c_{i j}$ are constants. Levin's inequality (1.3) shows that $I_{1}$ will be the union of all subintervals $\left[x_{j}, x_{j+1}\right]$ of $I$ such that $n_{j+1}+\cdots+n_{k}$ is odd and $I_{2}$ will be the union of all such subintervals such that the same sum is even.

(ii) Consider the $q$-focal BVP

$$
L y=y^{(n)}-\lambda p(x) y=f(x, y)
$$

with boundary conditions

$$
\begin{array}{ll}
y^{(i)}(a)=c_{i}, & i=0,1, \ldots, q-1, \\
y^{(j)}(b)=c_{j}, & j=q, \ldots, n-1,
\end{array}
$$


where $p(x)>0$ is continuous, $\lambda= \pm 1$ and the equation $L y=0$ is disfocal on $I$, i.e., has no nontrivial solution $y(x)$ such that each of the derivatives $y^{(k)}(x)$, $k=0,1, \ldots, n-1$, vanishes at least once in $I$ (see [3]). Peterson [5] has determined the Green's function for the associated homogeneous problem and has shown that its sign is determined by the inequality

$$
(-1)^{n-q} G(x, s)>0 \text { for all }(x, s) \in(a, b) \times(a, b) .
$$

Hence in this case $I=I_{1}$ if $n-q$ is odd; $I=I_{2}$ if $n-q$ is even.

REMARKs. (i) Because of the general form of the boundary conditions (2.2), we require that the initial approximants $v_{1}$ and $w_{1}$ satisfy the boundary conditions. For the conjugate $k$-point BVP, this requirement can be relaxed somewhat. A modification of the iteration (2.7) or (2.14) then leads to the conclusions of Theorem 1 or Theorem 2, if one begins with functions $v_{1}$ and $w_{1}$ satisfying the boundary conditions (3.1)-(3.4) of Theorem 3.1 of [6].

(ii) Theorems 1 and 2 remain valid under Carathéodory conditions, in the case of Theorem 1 under the hypothesis that (2.4) holds for $(x, y) \in I \times \mathbf{R}$ for almost all $x$. Theorem 2 extends a result of V. Seda [7] to the case of general linear boundary conditions.

(iii) For certain boundary problems, not only the sign of Green's function $G(x, s)$, but also the signs of some of its derivatives $\partial^{r_{p}} G(x, s) / \partial x^{r_{p}}, p=1, \ldots, p_{0}$, $r_{p}<n$, are independent of $s$ (see, for instance, [5]). (As an example, for the problem $y^{\prime \prime}=0, y(0)=a, y^{\prime}(1)=b, G(x, s) \leqslant 0, \partial G(x, s) / \partial x \leqslant 0$.) In such cases, Theorems 1 and 2 can be extended in a natural way to problems of the form $L y=f\left(x, y, y^{\left(r_{1}\right)}, \ldots, y^{\left(r_{p 0}\right)}\right), T y(x)=r$.

\section{REFERENCES}

1. W. A. Coppel, Disconjugacy, Lecture Notes in Math., vol. 220, Springer-Verlag, Berlin, 1971.

2. L. K. Jackson, Boundary value problems for ordinary differential equations, Studies in Ordinary Differential Equations (J. K. Hale, ed.), MAA Studies in Mathematics, vol. 14, Mathematical Association of America, Washington, D. C., 1977.

3. Z. Nehari, Disconjugate linear differential operators, Trans. Amer. Math. Soc. 129 (1967), 500-516.

4. A. C. Peterson, On the sign of Green's functions, J. Differential Equations 21 (1976), 167-178.

5. Green's functions for focal type boundary value problems, Rocky Mountain J. Math. (to appear).

6. K. Schmitt, Boundary value problems and comparison theorems for ordinary differential equations, SIAM J. Appl. Math. 26 (1974), 670-678.

7. V. Seda, Two remarks on boundary value problems for ordinary differential equations, J. Differential Equations 26 (1977), 278-290.

Department of Mathematics and Statistics, University of Nebraska-Lincoln, Lincoln, Nebraska 68588

Department of Mathematics, University of Missouri, Rolla, Missouri 65401 (Current address of both authors) 